\title{
Aplicação de Jogos Adaptativos na Educação: uma Revisão Sistemática da Literatura
}

\author{
Wendell Oliveira de Araújo ${ }^{1}$, Thiago Reis da Silva ${ }^{1,2}$, Eduardo H. S. Aranha ${ }^{1}$ \\ ${ }^{1}$ Programa de Pós-Graduação em Sistemas Computacionais - PPgSC \\ Universidade Federal do Rio Grande do Norte - UFRN \\ Laboratório Gamedu.net \\ ${ }^{2}$ Instituto Federal de Educação, Ciência e Tecnologia do Maranhão - IFMA \\ wendell.cmdegmail.com, thiago.reiseifma.edu.br, \\ eduardoaranhaedimap.ufrn.br
}

\begin{abstract}
Introduction: adaptive educational games emerge as a tool to help improving students learning. Objectives: to investigate how adaptive educational games are being applied in education, trying to discover what approaches and techniques are being used to create these games. Method: a systematic literature review was conducted to check the results of previously published works. Results: after application of the inclusion and exclusion criteria, of the 634 primary studies, 13 were included in this study. Conclusion: different approaches are being applied to develop adaptive educational games and they bring interesting benefits. In addition, the results confirm the lack of publications in the area.
\end{abstract}

Resumo. Introdução: os jogos educacionais adaptativos surgem como uma ferramenta para ajuda a melhorar o aprendizado dos alunos. Objetivos: investigar como os jogos educacionais adaptativos estão sendo aplicados na educação, procurando descobrir que abordagens e técnicas estão sendo utilizadas para criá-los. Método: foi realizada uma revisão sistemática da literatura, para buscar resultados de trabalhos publicados anteriormente. Resultados: após a aplicação dos critérios de inclusão e exclusão, dos 634 estudos primários, 13 foram incluidos neste estudo. Conclusão: diferentes abordagens estão sendo aplicadas para desenvolvê-los e os mesmos trazem benefícios interessantes. Além disso, os resultados permitiram confirmar a carência de publicações na área.

\section{Introdução}

Com o decorrer dos anos, é notável que os jogos venham ocupando cada vez mais espaço na área educacional. Isso ocorre por os jogos possuírem um grande potencial para o aprendizado [Brazil e Baruque 2015] e por ser algo que atrai o interesse dos estudantes. Existem vários jogos educativos disponíveis, porém, é difícil utilizar todo o potencial dos jogos para manter o conteúdo educativo e um jogo interessante para o aluno [Lopes e Bidarra 2011]. Neste sentido, a adaptabilidade dos jogos pode ajudar.

Jogos adaptativos são jogos que podem ser modificados durante a sua execução, podendo apresentar, entre outras coisas, novos desafios e/ou obstáculos, realizando adaptações nos níveis dificuldades, no cenário, de acordo com cada jogador. No contexto educacional, podemos utilizar esses jogos para que cada aluno aprenda novos conteúdos no seu ritmo, pois o jogo se adequará à dificuldade do jogador [Hwang et. al., 
V Congresso Brasileiro de Informática na Educação (CBIE 2016)

Anais do XXVII Simpósio Brasileiro de Informática na Educação (SBIE 2016)

2013]. À medida que um aluno está jogando, o nível de difículdade do conteúdo e do jogo pode ser modificado para que o jogo continue atrativo para o aluno. Um jogo adaptativo pode ser desenvolvido de várias formas e técnicas diferentes de acordo com a necessidade do jogo [Kickmeier e Albert 2012].

Visando identificar como os jogos adaptativos estão sendo aplicados na educação e quais técnicas estão sendo utilizadas para desenvolvê-los, realizamos uma Revisão Sistemática da Literatura (RSL). Uma RSL é uma pesquisa em profundidade de um fenômeno de interesse que produz resultados específicos e detalhados por meio da análise de conteúdo e qualidade do material pesquisado [Kitchenham 2004]. Para realizar a revisão proposta por este trabalho, foram utilizadas as recomendações apresentadas em Kitchenham (2004) e envolvendo as seguintes etapas: definição das questões de pesquisa, do processo de busca e critérios de inclusão e exclusão; da string de busca, da avaliação da qualidade, da coleta e análise de dados e do procedimento de distribuição e análise dos artigos.

Para apresentar o planejamento, a execução e os resultados do trabalho, o restante deste artigo segue organizado em quatro seções. A Seção 2 apresenta o método de pesquisa utilizado. Logo após, na Seção 3, os resultados e, por fim, na Seção 4 são feitas as considerações finais.

\section{Metodologia}

Esta RSL está estruturada com base nas diretrizes originais propostas por Kitchenham (2004). No caso desta revisão o objetivo foi o de analisar como os jogos adaptativos estão sendo aplicados na educação e quais técnicas principais estão sendo utilizadas para desenvolvê-los. Para esta RSL analisamos o cenário internacional entre os anos de 2011 a 2015, sendo incluídos artigos de 01 de janeiro de 2011 a 31 de dezembro de 2015 .

\subsection{Questões de Pesquisa}

Essa revisão visa responder as seguintes Questões de Pesquisa (QP), sendo elas:

- QP1: Quais abordagens estão sendo utilizadas para implementar a adaptabilidade dos jogos?

- QP2: Quais níveis de escolaridade estão sendo destinados esses jogos?

- QP3: Quais as áreas/disciplinas de conhecimento que estão sendo trabalhadas nos jogos?

- QP4: Existe relação entre os jogos trabalhados e o conteudo visto nas escolas?

- QP5: Quais os benefícios e limitações que estão sendo relatados pela aplicação dos jogos desenvolvidos?

\subsection{Processo de Busca, Critérios de Inclusão e Exclusão}

Considerando o objetivo da pesquisa envolvendo diversos domínios (educação, computação e jogos), selecionamos uma única ferramenta de busca que foi o Google Scholar. No contexto do artigo, a opção pela Google Scholar justifica-se porque os artigos publicados em revistas reconhecidas cientificamente são encontrados por este mecanismo de busca, mas também permite a busca de artigos publicados em outras 
V Congresso Brasileiro de Informática na Educação (CBIE 2016)

Anais do XXVII Simpósio Brasileiro de Informática na Educação (SBIE 2016)

fontes de pesquisas, como, repositórios digitais, por exemplo, IEEE e ACM e com isso tornam-se possível a busca em diversos meios e domínios de publicação on-line.

Uma abordagem em três etapas foi utilizada no processo de busca dos estudos. Na primeira etapa, todos os estudos primários recuperados foram avaliados a fim de se identificar aqueles relevantes para responder às questões de pesquisa. Um total de 634 estudos foram analisados. Após a leitura dos títulos, resumos e palavras chaves, este conjunto inicial foi reduzido para 23. Durante esta triagem inicial, foram aplicados os Critérios de Inclusão (CI) e Critérios de Exclusão (CE) vistos na Tabela 1.

Tabela 1: Critérios de inclusão e exclusão.

\begin{tabular}{|l|l|}
\hline \multicolumn{1}{|c|}{ Critérios de Inclusão } & \multicolumn{1}{c|}{ Critérios de Exclusão } \\
\hline CI1: Artigos completos que abordam uso & CE1: Ser um jogo adaptativo, porém, não \\
de jogos educacionais adaptativos; & educativo; \\
CI2: Relatam a implementação ou a a & CE2: Documentos que estão disponíveis \\
aplicação de algum estudo empírico ou & na forma de resumos ou apresentações e \\
técnica para o desenvolvimento e e & estudos secundários (ou seja, revisões \\
aplicação do jogo; & sistemáticas da literatura e mapeamentos \\
CI3: Está escrito em inglês; e & sistemáticos); \\
CI4: Publicações entre 2011 a 2015. & CE3: Não estiver escrito em Inglês; e \\
& CE4: Artigos derivados da mesma \\
& pesquisa (Se estudos duplicados, exclui-se \\
& o menos atual). \\
\hline
\end{tabular}

Em uma segunda etapa, procedeu-se à leitura dos resumos e conclusão e novamente à aplicação dos CI e CE nos 23 artigos candidatos e o resultado foi um subconjunto de 18 estudos primários. É importante ressaltar que, em alguns casos, a leitura de outras partes dos artigos foi realizada para se proceder à seleção. Com esses 18 artigos, foi feita uma análise mais profunda nos artigos e 5 artigos foram retirados por não se adequarem ao contexto da pesquisa, ficando assim 13 artigos para serem discutidos neste trabalho. Na terceira etapa, os 13 estudos que compõe a seleção final foram lidos na íntegra. As referências bibliográficas destes artigos podem ser visualizadas em: $<$ goo.gl/6nsjQv>.

\subsection{String de Busca}

String de busca é uma sequência de caracteres, com os termos principais, derivados das questões de pesquisa [Kitchenham 2004]. A string foi construída usando os operadores lógicos AND e OR, como mostrado a seguir:

\section{(“adaptive game” AND (education OR learning))}

Várias buscas foram executadas separadamente antes de se chegar a essa String, utilizando tanto buscas com todos os termos, quanto buscas mais amplas usando somente partes do termo da busca. Durante a busca encontramos artigos enquadrados nos termos, contudo, sem relação com a pesquisa. Por exemplo, sobre jogos adaptativos, mas sem o foco educacional. De fato, analisamos todas as 64 páginas dos resultados do Google Scholar. 
V Congresso Brasileiro de Informática na Educação (CBIE 2016)

Anais do XXVII Simpósio Brasileiro de Informática na Educação (SBIE 2016)

\subsection{Avaliação da Qualidade}

Definir e avaliar a qualidade pretendida para os estudos primários está além dos critérios de inclusão e exclusão [Kitchenham 2004]. Os critérios de qualidade ajudam a classificar detalhes após a inclusão, a orientar recomendações futuras e a interpretar melhor os resultados da pesquisa.

Este processo de Avaliação da Qualidade (AQ) foi realizado durante a fase de extração de dados e garantiu que estudos incluídos tivessem uma contribuição relevante para a RSL. Os critérios de AQ utilizados neste estudo são discutidos por Dyba e Dingsøyr (2008). Os sete critérios utilizados foram:

- AQ1: Existe uma definição clara dos objetivos da pesquisa?

- AQ2: Existe uma descrição adequada do contexto que a pesquisa foi realizada?

- AQ3: O planejamento da pesquisa foi adequado para abordar os objetivos da pesquisa?

- AQ4: A estratégia de extração dos dados foi adequada aos objetivos da pesquisa?

- AQ5: A análise dos dados foi suficientemente rigorosa?

- AQ6: Será que a relação entre pesquisador e participantes foi considerada em um grau adequado?

- AQ7: Existe uma indicação clara dos resultados?

Os dois primeiros critérios foram utilizados para excluir estudos que não indicam claramente os objetivos da pesquisa. Isto representa o limiar mínimo de qualidade que foi observada durante esta RSL. Os critérios restantes visam determinar o rigor, análise e credibilidade dos métodos de pesquisa empregados, bem como a relevância e a qualidade de cada estudo para esta revisão. As respostas para cada questão foram tabuladas e atribuídas um valor de 1 ("Sim") ou 0 ("Não"). A fim de testar a validade do procedimento de avaliação de qualidade, esta foi avaliada por dois revisores distintos. Não houveram discordâncias dos revisores em termos de avaliação da qualidade desses trabalhos.

\subsection{Procedimentos de Distribuição dos Artigos e de Coleta de Dados}

A partir da lista de publicações identificadas, os artigos foram atribuídos de forma aleatória pelo pesquisador doutor R3 para outros dois pesquisadores alunos de pósgraduação (R1 e R2). Cada pesquisador avaliou individualmente seus artigos e os resultados de R1 e R2 foram integrados por R3 na tabela de Concordância/Discordância. Por fim, R3 julgou e resolveu as discordâncias na tabela, numa lista final de estudos avaliados. Os pesquisadores R1 e R2 analisaram artigos distintos, ou seja, cada artigo foi lido por apenas um revisor.

Foram extraídos os seguintes dados sobre os estudos selecionados: título, evento/periódico, ano, autores, objetivos do estudo; como os dados foram coletados e analisados durante o estudo; escolaridade a qual a pesquisa foi aplicada (por exemplo, Ensino Médio ou Superior); disciplina e curso onde a pesquisa foi aplicada; os resultados, conclusões e dificuldades encontradas; e a avaliação de qualidade do estudo. 
V Congresso Brasileiro de Informática na Educação (CBIE 2016)

Anais do XXVII Simpósio Brasileiro de Informática na Educação (SBIE 2016)

Todos os dados foram extraídos pelos revisores, de forma independente. Estes resultados foram então comparados. Como não existiram diferenças significativas entre os resultados, a estratégia de extração de dados foi considerada apropriada. Todos os dados extraídos foram consolidados em uma planilha.

\section{Resultados}

Esta seção detalha os resultados do estudo. Assim, na Seção 3.1 será apresentada a avaliação da qualidade dos estudos primários. A Seção 3.2 por sua vez apresenta as respostas às questões de pesquisa. Na seção seguinte, são reportadas as limitações e ameaças do estudo e, finalmente, na Subseção 3.4, os trabalhos relacionados.

\subsection{Avaliação da Qualidade dos Estudos Reportados}

Os resultados desta avaliação da qualidade são apresentados na Tabela 2. Todos os artigos incluídos na revisão tiveram seus objetivos claramente definidos (QA1) e ofereceram alguma descrição do contexto em que a pesquisa foi realizada (QA2). Ainda, todos eles foram considerados como tendo um projeto de pesquisa apropriado (QA3).

A Tabela 2 apresenta a pontuação dos artigos nos critérios de qualidade. Ela mostra que os estudos incluídos tinham uma estratégia de extração adequada ao contexto da pesquisa (QA4), e que a análise dos dados foi considera suficientemente rigorosa em 10 dos artigos analisados (QA5). Já a relação entre o pesquisador e os participantes foi considerada adequada em nove estudos (QA6), e todos os estudos apresentaram uma indicação clara dos resultados (QA7). No final da avaliação, pudemos observar que nove estudos obtiveram a pontuação máxima de oito pontos [E1, E2, E3, E5, E6, E8, E9, E10 e E13]. Já a pontuação mais baixa que os artigos alcançaram foi de cinco pontos [E4, E7 e E12]. Finalmente, a pontuação média em qualidade dos trabalhos incluídos na revisão foi de 7,2 .

Tabela 2: Resultados da Avaliação da Qualidade.

\begin{tabular}{|c|c|c|c|c|c|c|c|c|}
\hline Estudos & QA1 & QA2 & QA3 & QA4 & QA5 & QA6 & QA7 & Total \\
\hline E1 & 1 & 1 & 1 & 1 & 1 & 1 & 1 & $\mathbf{8}$ \\
\hline E2 & 1 & 1 & 1 & 1 & 1 & 1 & 1 & $\mathbf{8}$ \\
\hline E3 & 1 & 1 & 1 & 1 & 1 & 1 & 1 & $\mathbf{8}$ \\
\hline E4 & 1 & 1 & 1 & 0 & 0 & 0 & 1 & $\mathbf{5}$ \\
\hline E5 & 1 & 1 & 1 & 1 & 1 & 1 & 1 & $\mathbf{8}$ \\
\hline E6 & 1 & 1 & 1 & 1 & 1 & 1 & 1 & $\mathbf{8}$ \\
\hline E7 & 1 & 1 & 1 & 0 & 0 & 0 & 1 & $\mathbf{5}$ \\
\hline E8 & 1 & 1 & 1 & 1 & 1 & 1 & 1 & $\mathbf{8}$ \\
\hline E9 & 1 & 1 & 1 & 1 & 1 & 1 & 1 & $\mathbf{8}$ \\
\hline E10 & 1 & 1 & 1 & 1 & 1 & 1 & 1 & $\mathbf{8}$ \\
\hline E11 & 1 & 1 & 1 & 0 & 1 & 0 & 1 & $\mathbf{6}$ \\
\hline E12 & 1 & 1 & 1 & 0 & 0 & 0 & 1 & $\mathbf{5}$ \\
\hline E13 & 1 & 1 & 1 & 1 & 1 & 1 & 1 & $\mathbf{8}$ \\
\hline Total & $\mathbf{1 3}$ & $\mathbf{1 3}$ & $\mathbf{1 3}$ & $\mathbf{9}$ & $\mathbf{1 0}$ & $\mathbf{9}$ & $\mathbf{1 3}$ & $/$ \\
\hline
\end{tabular}


V Congresso Brasileiro de Informática na Educação (CBIE 2016)

Anais do XXVII Simpósio Brasileiro de Informática na Educação (SBIE 2016)

\subsection{Respostas as Questões de Pesquisa}

Nesta subseção, apresentam-se as respostas às questões de pesquisa desta RSL. Um resumo das informações que foram extraídos de cada estudo incluído pode ser encontrado em: < goo.gl/vyd6yA $>$.

\section{QP1: Quais abordagens estão sendo utilizadas para implementar a adaptabilidade dos jogos?}

Ao analisar os estudos selecionados, nota-se que as abordagens utilizadas para a adaptação dos jogos são variadas. Dos trabalhos selecionados, três trabalhos faziam o uso de métodos ou estilos de aprendizagem, formas de como o aprendizado pode ser realizado. Um utilizou a técnica de Scaffolding [E1]. Outro utilizou Aprendizagem Espaçada, a qual separam momentos de aprendizado intensivo e "diversão" em blocos de tempo para o mesmo ir aprendendo e se divertindo [E2]. Já no estudo [E9], o jogo detectava o estilo de aprendizagem do jogador e aplicava a mudança no jogo para se adequar a ele.

Outros três trabalhos [E4, E5 e E11] utilizaram técnicas de Inteligência Artificial para criar jogos adaptativos. Em [E5], os autores abordam o Aprendizado por Reforço, Redes Neurais e Algoritmo Genético para a adaptação do jogo. Já [E11] faz uso de Redes Neurais e [E4] aborda o uso de técnicas de Aprendizado de Máquina.

Uma técnica também destacada pelos trabalhos [E3, E6 e E7] é o uso de Computação Afetiva, o qual tenta adaptar as condições do jogo e níveis de dificuldade baseadas no reconhecimento da emoção do jogador e encontrando maneiras de deixá-lo mais motivado. Alguns trabalhos também fizeram o uso de Modelagem do Jogador ou usuário para adaptar o jogo, fazendo uma coleta de dados do jogador à medida que ele jogava ou através de questionários ou capturando informações de outros lugares para adequar o jogo ao jogador [E8, E10 e E12]. Já [E13] informa que dois tipos de adaptabilidades foram implementados no jogo. A micro adaptabilidade, para fornecer um incentivo motivacional e dicas através de um personagem não jogável, e a macro adaptabilidade, alterando o ritmo da história do jogo. A Figura 1 apresenta um resumo das técnicas utilizadas nos estudos.

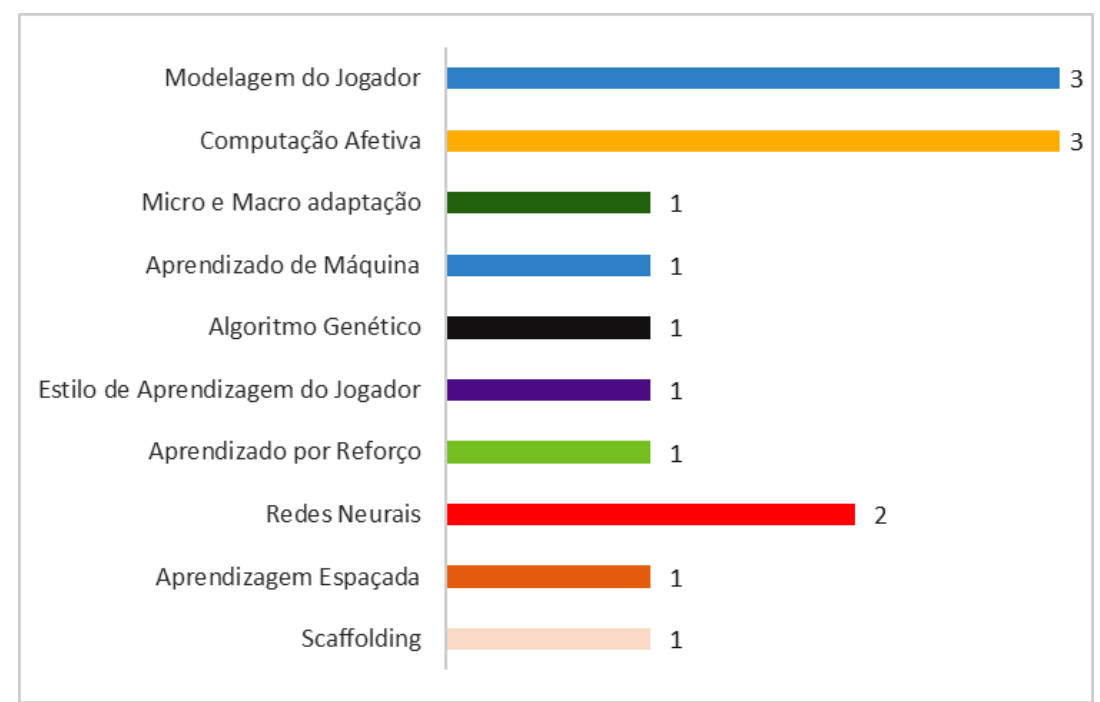

Figura 1: Técnicas utilizadas para desenvolver jogos educacionais adaptativos. 
V Congresso Brasileiro de Informática na Educação (CBIE 2016)

Anais do XXVII Simpósio Brasileiro de Informática na Educação (SBIE 2016)

\section{QP2: Quais níveis de escolaridade estão sendo destinados esses jogos?}

Os artigos foram classificados de acordo com o nível de escolaridade para os quais as pesquisas se destinaram. Observado a Figura 2 é possível perceber que os jogos estão sendo destinado em sua maioria para o Ensino Fundamental II (do $6^{\circ}$ ao $9^{\circ}$ ano), correspondendo a 39\% [E1, E4, E5, E6 e E13]. Em contraste, 23\% tem foco no Ensino Fundamental I (do $1^{\circ}$ ao $5^{\circ}$ ano) [E3, E8 e E10] e 23\% das pesquisas se destinam ao Ensino Superior [E2, E7 e E9]. Destes, 15\% [E11 e E12] não informaram para quais níveis estavam sendo destinadas os estudos.

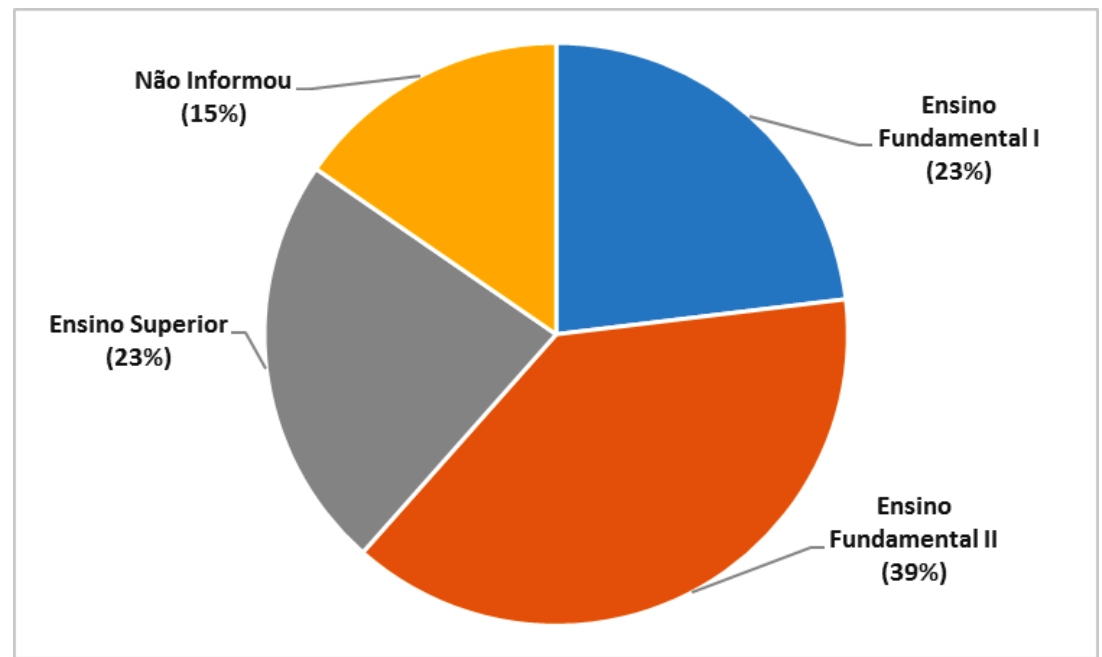

Figura 2: Nível de escolaridade a qual os jogos estão sendo destinados.

QP3: Quais as áreas/disciplinas de conhecimento que estão sendo trabalhadas nos jogos?

Houve uma variação nos conteúdos ao quais os jogos estavam sendo desenvolvidos. Dois jogos foram desenvolvidos para Matemática [E8 e E10], um para ensino de Banco de Dados [E9], outro para Gestão de Projetos [E7], outro para Microbiologia [E6], um para Resolução de Conflitos [E5], um para Espanhol [E1], um para o ensino de Chinês [E3], um para Geografia [E13] e outro para Medicina [E2]. No entanto, três estudos [E4, E11 e E12] não citaram ou apresentaram uma disciplina específica a ser utilizada. A Figura 3 ilustra essa distribuição.

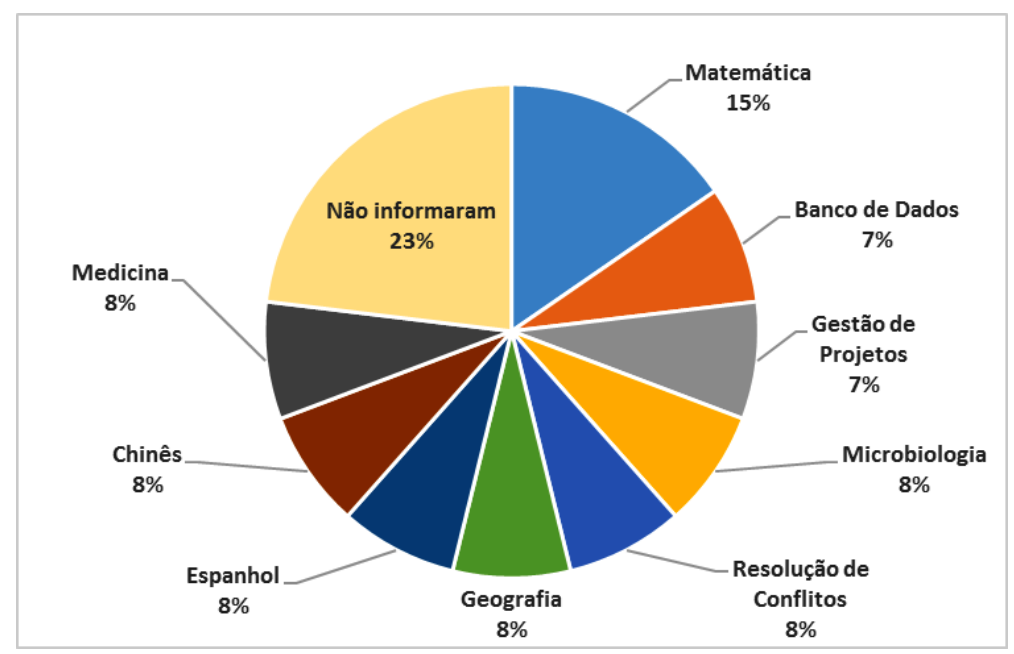

Figura 3: Disciplinas reportadas nos estudos. 
V Congresso Brasileiro de Informática na Educação (CBIE 2016)

Anais do XXVII Simpósio Brasileiro de Informática na Educação (SBIE 2016)

\section{QP4: Existe relação entre os jogos trabalhados e o conteúdo visto nas escolas?}

Como os jogos estão sendo desenvolvidos para o âmbito educacional, é necessário saber se o conteúdo do jogo possuía relação com os conteúdos vistos em salas de aulas. Os estudos [E1, E2, E3, E6, E8, E9, E10 e E13] abordavam jogos com conteúdos vistos em sala de aula. Os trabalhos [E11 e E12] não descreveram se tinham relação com o conteúdo da sala de aula. O trabalho [E5] não abordava o conteúdo da sala de aula, pois ele trabalhava com resolução de conflitos. Os demais trabalhos não apresentaram relação entre o propósito do jogo e conteúdo visto em sala.

\section{QP5: Quais os benefícios e limitações que estão sendo relatados pela aplicação dos jogos desenvolvidos?}

O propósito desta questão é averiguar que tipos de benefícios esses jogos podem trazer. Com a análise dos estudos, todos os trabalhos relataram benefícios no uso de jogos, dos quais oito deles [E3, E4, E6, E7, E8, E9, E10 e E13] apontaram como o principal benefício no uso dos jogos a motivação gerada, pois os alunos ficaram mais interessados em participar desse tipo de atividade.

Os trabalhos [E8 e E10] também apontam que os jogos são uma ótima maneira de testar os alunos, realizando a coleta de seus dados à medida que ele vai jogando. Além disso, os trabalhos [E1, E2, E5, E6 e E9] também destacaram que o aprendizado dos alunos foi mais eficiente utilizando os jogos adaptativos do que através de meios tradicionais ou jogos não adaptativos. Apenas [E13] relatou uma limitação na aplicação do jogo, pois quando a competência do jogador era maior do que o desafio proposto pelo jogo, eles achavam uma resolução trivial e "chata", relatando que o jogo não adaptou adequadamente para esse jogador. Os demais estudos não apresentaram as limitações.

\subsection{Ameaças a Validade da RSL}

No geral, as principais ameaças à validade da RSL são em relação ao viés de seleção de publicações e aos dados imprecisos extraídos. As pesquisas foram concebidas empregando principalmente recursos eletrônicos. Estes foram pesquisados após a implementação de pesquisas experimentais, strings de busca e utilização de dicionários de sinônimos. Apesar disso, não é possível garantir que todos os estudos relevantes foram coletados e há um pequeno risco de que alguns estudos podem ter sido omitidos, devido aos termos de busca utilizados. O processo de extração de dados também pode resulta em dados irrelevantes. Isto pode ocorre, porque o processo de extração de dados foi realizado somente por dois pesquisadores. O desenvolvimento de um protocolo da RSL e o uso de uma estratégia de verificação da qualidade da revisão (por um terceiro revisor) ajuda a garantir que este não foi o caso. Finalmente, é possível que os critérios de inclusão possam, ocasionalmente, terem excluídos alguns estudos relevantes. Isso pode ocorrer porque os critérios implementados nos estudos não continham palavraschaves apresentadas nos títulos ou nos resumos. Esse risco foi minimizado através da não remoção dos artigos que gerassem dúvidas.

\subsection{Trabalhos Relacionados}

Pietruchinski et. al., (2011) desenvolveram e publicaram um estudo sobre os jogos educativos no contexto do Simpósio Brasileiro de Informática na Educação (SBIE). Para 
V Congresso Brasileiro de Informática na Educação (CBIE 2016)

Anais do XXVII Simpósio Brasileiro de Informática na Educação (SBIE 2016)

tal, utilizaram uma metodologia de RSL visando extrair, catalogar, analisar e sintetizar dados de todos os artigos publicados em 10 edições (2001-2010) do SBIE. Silva, Aranha e Medeiros (2014) apresentam os resultados de uma RSL de jogos digitais para ensino de programação. Nessas duas pesquisas não foram encontrados e nem reportados o uso de jogos adaptativos.

Em contrapartida, Ferreira e Ishitani (2015) em sua RSL sobre jogos digitais para idosos relata vários jogos, sendo um deles adaptativo. Da mesma forma, outra pesquisa mapeou um cenário mais específico dentro da temática deste trabalho. Pituba e Nakamura (2014) apresentou uma RSL para avaliar as estratégias de adaptação de jogos adaptativos. Esses encontraram um total de 12 projetos em 16 estudos, onde 8 foram testados apesar de alguns não mostrarem grandes detalhes dos testes. Outro trabalho é focado em dois grandes projetos com adaptação. Este estudo desenvolveu um processo similar ao apresentado por Pituba e Nakamura (2014), aplicando a técnica de pesquisa secundária (a RSL) para realizar uma análise abrangente e sistemática da pesquisa em jogos adaptativos com o foco educacional.

\section{Considerações Finais}

Este estudo teve como objetivo aplicar o método da RSL para determinar e qualificar os estudos existentes sobre jogos educacionais adaptativos nos últimos cincos anos em um cenário internacional. Foram encontrados inicialmente 634 artigos e após a análise dos mesmos, 13 foram incluídos para extração dos dados. Os jogos educacionais adaptativos são desenvolvidos com o propósito de melhorar o rendimento dos alunos, aumentar o interesse dos alunos em jogá-los. Segundo os resultados da RSL, o uso desses jogos aumentou o interesse dos alunos em participar desse tipo de atividade e que o aprendizado dos alunos foi mais eficiente que os meios tradicionais.

Cinco questões de pesquisa foram criadas com o objetivo de verificar o quão efetivo e o uso dos jogos educacionais adaptativos e que técnicas estão sendo utilizadas para desenvolvê-los. Vários resultados puderam ser extraídos dos dados recolhidos dos estudos. Com eles, pudemos observar que (i) a computação afetiva e a modelagem do jogador são as abordagens que foram mais frequentemente usadas nas pesquisas; (ii) os jogos estão sendo mais aplicado no ensino fundamental II e nos estudos não foram encontradas aplicações no ensino médio; (iii) não há um foco específico em uma disciplina específica para desenvolver esses jogos, pois encontramos jogo trabalhando diferentes disciplinas.

Os resultados tabulados e discutidos permitiram concluir que o método da RSL identificou a carência de publicações sobre jogos educacionais adaptativos e permitiu selecionar e qualificar estudos que ajudaram a responder parcialmente as questões de pesquisa. Podemos reportar que não foram encontrados artigos sobre jogos adaptativos para o público do ensino médio. Os resultados demonstram também que a área de jogos educacionais adaptativos tem muito potencial para evoluir e, pelos relatos encontrados, eles possuem efetividade em seu propósito de motivar e melhorar o aprendizado dos alunos, fato este relatado em todos os estudos analisados. 
V Congresso Brasileiro de Informática na Educação (CBIE 2016)

Anais do XXVII Simpósio Brasileiro de Informática na Educação (SBIE 2016)

\section{Agradecimentos}

Os autores agradecem a CAPES pelo suporte parcial a esta pesquisa, ao Instituto Nacional de Engenharia de Software (INES), ao CNPq, aos integrantes do Laboratório Gamedu.net e, em especial, ao Programa de Pós-Graduação em Sistemas Computacionais - PPgSC/UFRN, por toda infraestrutura oferecida.

\section{Referências}

Brazil, A. L.; Baruque, L. B. (2015) "Gamificação Aplicada na Graduação em Jogos Digitais”. In: XXVI Simpósio Brasileiro de Informática na Educação - SBIE, p.677686.

Dybå, T.; Dingsøyr, T. (2008) "Empirical Studies of Agile Software Development: A Systematic Review”. In: Information and Software Technology, v.51, p. 833-859.

Ferreira, R. L. R.; Ishitani, L. (2015) "Jogos educacionais digitais para idosos: uma revisão sistemática de literatura”. In: XXVI Simpósio Brasileiro de Informática na Educação - SBIE, p.404-413.

Hwang; G-J.; Sung; H-Y.; Hung, C.-M.; Huang, I. (2013) “A Learning Style Perspective to Investigate the Necessity of Developing Adaptive Learning Systems". In: Journal of Educational Technology and Society, v.16, p.188-197.

Kickmeier-Rust, M. D., Albert, D. (2012) "Educationally Adaptive: Balancing Serious Games". International Journal of Computer Science and Sport 11(1).

Kitchenham, B. (2004) "Procedures for performing systematic reviews". Tech. Report TR/SE-0401, Keele University, Keel, UK.

Lopes, R., Bidarra, R. (2011) "Adaptivity Challenges in Games and Simulations: A Survey". In: IEEE Transactions on Computational Intelligence and AI in Games, 3 (2), p.85-99.

Pietruchinski, M. H.; Coelho Neto, J.; Malucelli, A.; Reinehr, S. (2011) "Os jogos educativos no contexto do SBIE: uma revisão sistemática de Literatura". In: XXII Simpósio Brasileiro de Informática na Educação - SBIE, p.476-485.

Pituba, J. L.; Nakamura, R. (2014) "Assessing the strategies of adaptation of adaptive educational games". In: Conferência de Ciências e Artes dos Videojogos VIDEOJOGOS.

Silva, T. R.; Medeiros, T. J.; Aranha, E. H. S. (2014) "Jogos Digitais para Ensino e Aprendizagem de Programação: uma Revisão Sistemática da Literatura". In: XXV Simpósio Brasileiro de Informática na Educação - SBIE, p.692-701. 